\title{
Effect of disorder on transverse domain wall dynamics in magnetic nanostrips
}

\author{
Ben Van de Wiele, ${ }^{1}$ Lasse Laurson, ${ }^{2}$ and Gianfranco Durin ${ }^{3,4}$ \\ ${ }^{1}$ Department of Electrical Energy, Systems and Automation, Ghent University, B-9000 Ghent, Belgium \\ ${ }^{2}$ COMP Centre of Excellence, Department of Applied Physics, Aalto University, P.O. Box 14100, FIN-00076 Aalto, Finland \\ ${ }^{3}$ ISI Foundation, Via Alassio 11/c, I-10126 Torino, Italy \\ ${ }^{4}$ Istituto Nazionale di Ricerca Metrologica, Strada delle Cacce 91, I-10135 Torino, Italy
}

(Received 29 June 2012; published 24 October 2012)

\begin{abstract}
We study the effect of disorder on the dynamics of a transverse domain wall in ferromagnetic nanostrips, driven either by magnetic fields or spin-polarized currents, by performing a large ensemble of graphics processing unit-accelerated micromagnetic simulations. Disorder is modeled by including small, randomly distributed nonmagnetic voids in the system. Studying the domain wall velocity as a function of the applied field and current density reveals fundamental differences in the domain wall dynamics induced by these two modes of driving: For the field-driven case, we identify two different domain wall pinning mechanisms, operating below and above the Walker breakdown, respectively, whereas for the current-driven case pinning is absent above the Walker breakdown. Increasing the disorder strength induces a larger Walker breakdown field and current, and leads to decreased and increased domain wall velocities at the breakdown field and current, respectively. Furthermore, for adiabatic spin-transfer torque, the intrinsic pinning mechanism is found to be suppressed by disorder. We explain these findings within the one-dimensional model in terms of an effective damping parameter $\alpha^{*}$ increasing with the disorder strength.
\end{abstract}

DOI: $10.1103 /$ PhysRevB.86.144415

PACS number(s): 75.78.Fg, 72.25.Ba, 75.78.Cd

Domain wall (DW) dynamics in nanoscale ferromagnetic wires and strips driven by magnetic fields or spin-polarized currents is a subject of major technological importance for the operation of potential future nanoscale magnetic memory ${ }^{1,2}$ and $\operatorname{logic}{ }^{3}$ devices. In these devices information is typically stored as magnetic domains along a nanostrip or wire and is processed by DW motion. For the reliable operation of such devices it is of fundamental importance to understand and control the effect of imperfections or disorder on the DW dynamics, necessarily present in any realistic samples, e.g., in the form of thickness fluctuations and grain structure of the sample, or various impurities and defects in the material. At the same time, such systems constitute a low-dimensional limit of the general problem of driven elastic manifolds in a random potential. ${ }^{4}$

While the crucial importance of disorder for the dynamics of higher-dimensional DWs is well established, resulting in phenomena such as the Barkhausen effect, ${ }^{5}$ a majority of studies of DW motion in systems with nanostrip or wire geometry neglect disorder effects. This applies to both theoretical studies and interpretations of experimental results. Some exceptions include studies demonstrating enhanced DW propagation due to the roughness of the edges of the strip. ${ }^{6,7}$ Recently also the effect of spatially varying saturation magnetization $M_{s}$ on the dynamics of vortex walls was studied, resulting in an effective damping increasing with the disorder strength. ${ }^{8}$ Similar spatially distributed disorder has also been studied in a simplified, line-based model of a transverse DW. ${ }^{9,10}$ Experimental studies of DW dynamics in wires have revealed its stochastic nature in the case of short current pulses, ${ }^{11}$ and has been attributed to the presence of disorder in the samples, in combination with thermal effects. For longer current pulses, the resulting average DW velocities have been shown to be quite low, ${ }^{12}$ likely due to pinning effects induced by structural disorder. Dynamical pinning effects have also been observed in experiments of field-driven vortex wall dynamics. ${ }^{13,14}$ However, despite these advances, many details of the disorder effects on DW dynamics in nanostructures remain to be clarified.

In this paper, we consider by micromagnetic simulations the effect of disorder on the field and current-driven dynamics of a transverse DW in a narrow and thin permalloy strip. Disorder is modeled by including randomly positioned small nonmagnetic regions (voids) in the system. Our results show that the field- and current-driven DW dynamics exhibit remarkable differences which are only revealed in the presence of disorder. In particular, we identify two fundamentally different DW pinning mechanisms acting in a field-driven system, operating below and above the Walker breakdown field, respectively, with the latter mechanism being absent in the current-driven case. Also the Walker breakdown itself is affected by the presence of disorder, such that it is shifted to larger field and current values with increasing disorder strength. At the same time, the DW velocities at the breakdown field and current get smaller and larger, respectively. Furthermore, for adiabatic spin-transfer torque, the intrinsic pinning mechanism is found to be suppressed by disorder. These findings emphasize the importance of understanding the interplay between disorder, the DW structure, and the properties of the external driving force, and are shown to be related to an effective damping parameter $\alpha^{*}$ increasing with the disorder strength.

We perform a large ensemble of micromagnetic simulations with the graphics processing unit (GPU)-based micromagnetic simulator MuMax, ${ }^{15}$ making it possible to obtain large statistics for averaging over the disorder realizations. To study the time evolution of the magnetization $\mathbf{M}(\mathbf{r}, t)$ with an amplitude $M_{s}$, we solve the Landau-Lifshitz (LL) equation with the 
spin-transfer torque terms, ${ }^{16}$

$$
\begin{aligned}
\frac{\partial \mathbf{M}}{\partial t}= & -\frac{\gamma}{1+\alpha^{2}} \mathbf{M} \times \mathbf{H}_{\mathrm{eff}}-\frac{\alpha \gamma}{M_{s}\left(1+\alpha^{2}\right)} \mathbf{M} \times\left(\mathbf{M} \times \mathbf{H}_{\mathrm{eff}}\right) \\
& -\frac{b_{j}}{M_{s}^{2}\left(1+\alpha^{2}\right)} \mathbf{M} \times[\mathbf{M} \times(\mathbf{j} \cdot \nabla) \mathbf{M}] \\
& -\frac{b_{j}}{M_{s}\left(1+\alpha^{2}\right)}(\xi-\alpha) \mathbf{M} \times(\mathbf{j} \cdot \nabla) \mathbf{M}
\end{aligned}
$$

where $\mathbf{H}_{\text {eff }}$ is the effective magnetic field (with contributions from the external, exchange, and demagnetization fields), $\gamma$ is the gyromagnetic ratio, $\alpha$ is the Gilbert damping constant, $\xi$ is the degree of nonadiabaticity, $\mathbf{j}$ is the current density, and $b_{j}=P \mu_{B} /\left[e M_{s}\left(1+\xi^{2}\right)\right]$, with $P$ the polarization, $\mu_{B}$ the Bohr magneton, and $e$ the electron charge.

We consider permalloy strips of width $w=100 \mathrm{~nm}$ and thickness $10 \mathrm{~nm}$, such that the stable DW structure is a head-tohead V-shaped symmetric transverse wall, separating in-plane domains pointing along the strip axis. ${ }^{17}$ The used material parameters are those of permalloy, i.e., $M_{s}=860 \times 10^{3} \mathrm{~A} / \mathrm{m}$ and $\alpha=0.02$, and no anisotropy fields are included in Eq. (1). To clearly see the effect of quenched disorder on the DW dynamics, we set the temperature $T=0$. The system is discretized by considering $N$ cells of size $3.125 \times 3.125 \times$ $10 \mathrm{~nm}^{3}$. Upon application of an external magnetic field $\mathbf{H}_{\text {ext }}=H_{\text {ext }} \hat{\mathbf{x}}$ along the strip axis in the absence of disorder, the DW is displaced along the strip. If the field is below the Walker breakdown field $H_{W}$, the DW essentially keeps its equilibrium structure during the propagation, with a small out-of-plane component close to the tip of the $\mathrm{V}$ shape, and a velocity roughly linearly proportional to the applied field. Above $H_{W}$, an antivortex is nucleated at the tip of the $\mathrm{V}$ shape. It then propagates across the strip width, reversing the polarity of the DW magnetization. This process is repeated such that the DW polarity oscillates back and forth, dramatically decreasing the average DW velocity. ${ }^{18}$

With disorder included in the form of randomly positioned nonmagnetic voids of linear size $3.125 \mathrm{~nm}$ with varying densities $\sigma$ within a strip of length $L=3.2 \mu \mathrm{m}$, the DW can get pinned even for nonzero applied fields. ${ }^{19}$ This makes measurement and even definition of the DW velocity a nontrivial task. Thus, in what follows we consider both the "conditional velocities" $v_{m}$ of the moving DWs, conditioned on the fact that the DWs will not get pinned during the time interval $\Delta t=20 \mathrm{~ns}$ we consider in the simulations (i.e., the DW will either reach the end of the strip or it is still moving after $\Delta t=20 \mathrm{~ns}),{ }^{20}$ and the probability $P_{\text {pin }}$ for the DW to get pinned during $\Delta t$. These are computed by averaging over 50 disorder realizations for each $H_{\text {ext }}$ and $\sigma$. Notice that here we consider a $T=0$ system, such that a pinned DW cannot depin. An alternative measure of the DW velocity (which is likely to be closer to typical experimental measurements where $T>0$ ) is given by $v_{\text {exp }}=\left(1-P_{\text {pin }}\right) v_{m}$. In general, $P_{\text {pin }}$ will increase with the observation (time and length) scale, thus making also $v_{\text {exp }}$ a scale-dependent quantity.

Figure 1 shows the resulting average velocities $v_{m}$ of the moving DWs as a function of $H_{\mathrm{ext}}$ and $\sigma$. The presence of voids induces a finite depinning field $H_{\text {dep }}(\sigma)$ increasing with $\sigma$. For $H_{\text {ext }}>H_{\text {dep }}(\sigma), v_{m}$ first increases until a maximum velocity is reached at $H_{\mathrm{ext}}=H_{W}(\sigma)$, and then starts to decrease

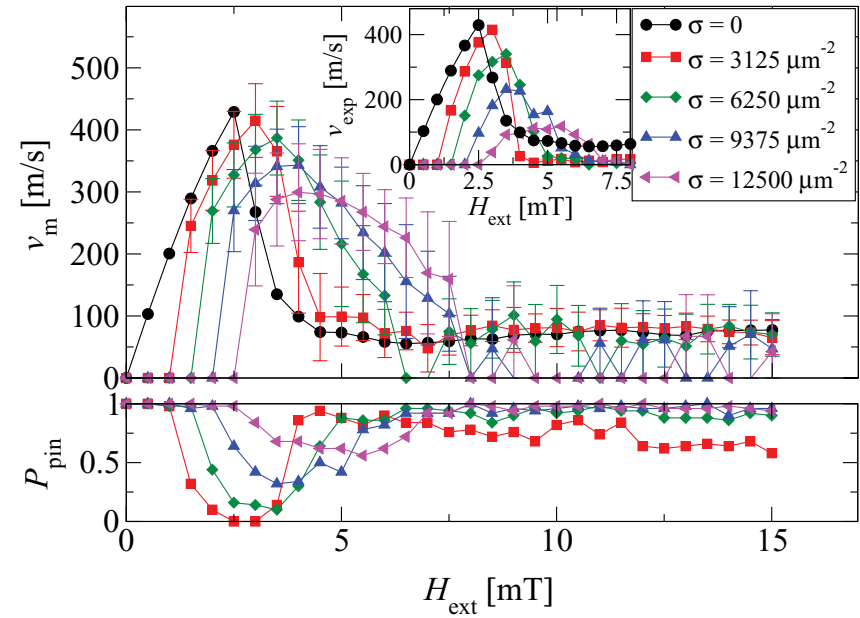

FIG. 1. (Color online) The average velocity $v_{m}$ of the moving DWs (main figure) and $v_{\text {exp }}=\left(1-P_{\text {pin }}\right) v_{m}$ (inset) as a function of $H_{\text {ext }}$ and $\sigma$. Error bars correspond to the standard deviation of $v_{m}$. The pinning probabilities $P_{\text {pin }}$ during the $20 \mathrm{~ns}$ simulation (bottom panel) exhibit large values for large $H_{\text {ext }}$ due to the core pinning mechanism.

again. The position $H_{W}(\sigma)$ of this maximum, corresponding to the Walker breakdown, is shifted towards larger field values as $\sigma$ is increased, and the corresponding maximum velocity $v_{m}\left[H_{W}(\sigma)\right]$ decreases with $\sigma$. The error bars in Fig. 1 correspond to the standard deviation of $v_{m}$, and indicate that the dynamics of moving DWs has a stochastic nature due to the random disorder. Notice in particular that the pinning probability $P_{\text {pin }}$ exhibits a nonmonotonic dependence on $H_{\text {ext }}$, with strong pinning for both small and large $H_{\text {ext }}$, while for intermediate applied fields (corresponding to large values of $v_{m}$ ) pinning is less likely. The maximum value of $v_{\exp }$ (inset of Fig. 1) exhibits a strong dependence on $\sigma$, and depends also on the observation scale via $P_{\text {pin }}$ (not shown). For large $H_{\text {ext }}, P_{\text {pin }}$ is close to 1 for $\Delta t=20 \mathrm{~ns}$, and consequently $v_{\text {exp }}$ is essentially zero. Similar pinning effects for large applied fields have been observed experimentally for vortex walls. ${ }^{13,14}$

To gain insight on the mechanisms behind this behavior, we consider snapshots of the DW configurations and the various contributions to $\partial \mathbf{M} / \partial t$ in Eq. (1). For small $H_{\text {ext }}$, we find that the overall DW structure is preserved, with the disorder inducing only minor distortions. If the DW gets pinned, this happens by a collective action of several voids. This mechanism is known as collective pinning, and it is responsible for the nonzero depinning field $H_{\mathrm{dep}}<H_{W}(\sigma)$. Remarkably, we identify a fundamentally different pinning mechanism for large fields, $H_{\mathrm{ext}}>H_{W}(\sigma)$ : In this regime, an antivortex is able to propagate to the interior of the strip, resulting in pinned DW configurations (occurring with probability $P_{\text {pin }}$ ) with the antivortex core positioned exactly on top of a void or a local void structure. We refer to this mechanism as core pinning, and attribute it to the fact that the energy of the system can be significantly lower when the antivortex core or part of it-involving large magnetization gradients and out-of-plane magnetization-is placed in a nonmagnetic region (or more generally, in a region with low $M_{s}$ ). In the field-driven case the DW is susceptible to get pinned by this mechanism because the Zeeman torque is relatively small in 


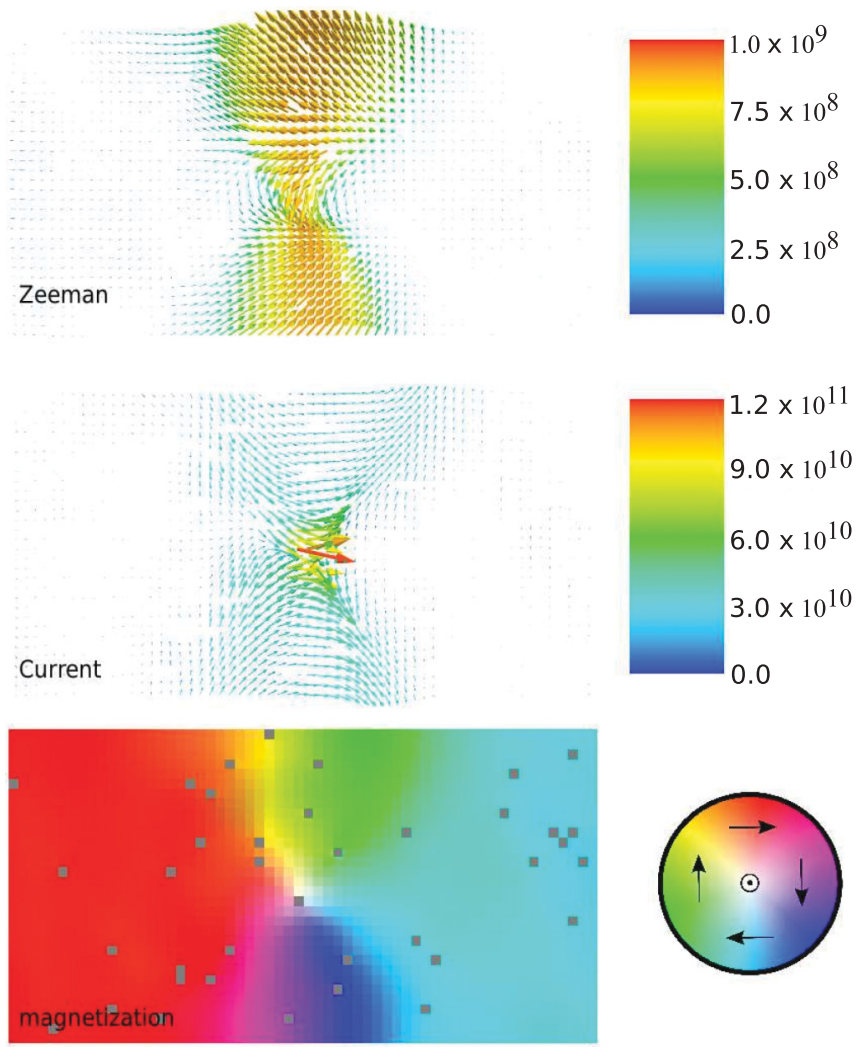

FIG. 2. (Color online) Examples of the spatial distribution of the contributions of the applied field $H_{\text {ext }}=5 \mathrm{mT}$ (top) and current density $j_{\mathrm{ext}}=20 \times 10^{12} \mathrm{~A} / \mathrm{m}^{2}$ with $\xi=0$ (middle) to $\partial \mathbf{M} / \partial t$ in Eq. (1), corresponding to the magnetization configuration shown in the bottom panel, exhibiting an antivortex in the middle of the strip. $\partial \mathbf{M} / \partial t$ is given in units of $M_{s} / \mathrm{s}$. The randomly positioned voids with $\sigma=3125 \mu \mathrm{m}^{-2}$ are shown as gray dots.

magnitude and does not directly displace the DW (top panel of Fig. 2); instead, the small out-of-plane magnetization due to the Zeeman torque induces demagnetizing fields, which act to move the DW. Such an indirect driving mechanism is sensitive to the perturbations due to disorder, leading to several effects, including $\sigma$-dependent $H_{\text {dep }}$ and $H_{W}$, and in particular the core pinning mechanism for high $H_{\text {ext }}$.

We proceed to contrast these results with the current-driven case by applying a current density $\mathbf{j}=-j_{\text {ext }} \hat{\mathbf{x}}$ with $P=0.5$ along strips of length $L=6.4 \mu \mathrm{m}$. We first consider perfect adiabaticity ( $\xi=0$, top panel of Fig. 3). Due to intrinsic pinning, ${ }^{21}$ there is a nonzero depinning current $j_{\text {dep,int }}$ in the absence of disorder, above which DW motion involves repeated polarity transformations mediated by antivortex propagation across the strip width. Adding disorder with the same procedure as above reveals two intriguing observations: First, it appears that the DW is able to move even for currents slightly below $j_{\text {dep,int }}$. This surprising finding can be explained by noticing that the intrinsic pinning mechanism is due to the ability of the DW to deform in such a way that the torques due to interactions within the DW (i.e., the effective field) exactly counterbalance the adiabatic spin-transfer torque. ${ }^{21}$ However, the presence of disorder induces additional DW deformations and imposes constraints on the ability of the DW to counteract the current-induced torques, leading to nonzero values for both
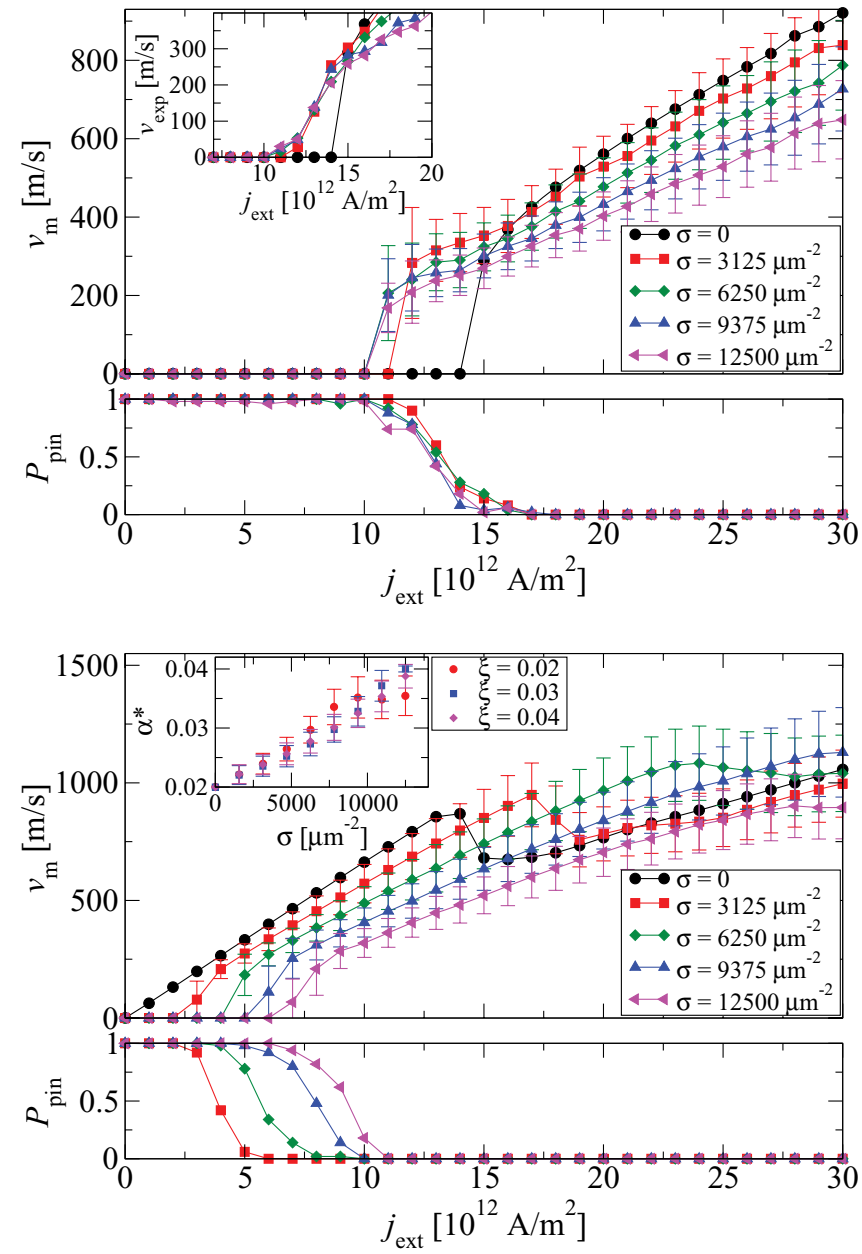

FIG. 3. (Color online) The average velocity $v_{m}$ of the moving DWs as a function of $j_{\mathrm{ext}}$ and $\sigma$, for $\xi=0$ (top) and $\xi=0.04$ (bottom). Error bars correspond to the standard deviation of $v_{m}$. The pinning probabilities $P_{\text {pin }}$ during the 20 ns simulation highlight the absence of core pinning for large current densities. The insets show $v_{\text {exp }}=\left(1-P_{\text {pin }}\right) v_{m}$ for $\xi=0$ (top panel), and the effective $\alpha^{*}(\sigma)$ for various $\xi$ (bottom panel), respectively.

$v_{m}$ and $1-P_{\text {pin }}$ for $j_{\text {ext }}$ somewhat below $j_{\text {dep,int }}$. Notice that while $v_{\text {exp }}$ (inset of the top panel in Fig. 3) exhibits nonlinear field dependence reminiscent of typical creep motion for small fields, we are considering here a $T=0$ system in which a pinned DW cannot depin due to the absence of thermal fluctuations. $^{22}$

The second observation is that for larger $j_{\text {ext }}$, core pinning is absent. Even if for $j_{\mathrm{ext}}>j_{W}(\sigma)$ the antivortex core is constantly moving back and forth across the strip width, it never gets pinned by the voids, strongly contrasting with the field-driven case. To explain this observation, we consider the spatial distribution of the current-induced contribution to $\partial \mathbf{M} / \partial t$ (middle panel of Fig. 2), and find that the current acts directly (in contrast to the indirect mechanism in the field-driven case) and strongly on the antivortex core where the magnetization gradients are large, facilitating its propagation along the strip across the energy barriers due to the voids. This is also directly visible in the the LL equation [Eq. (1)], where the current acts on the gradient of $\mathbf{M}$ rather than on $\mathbf{M}$ itself. 
TABLE I. Predictions for $j_{\text {dep,int }}, H_{W}$, and $v_{m}\left(H_{W}\right)$ from the one-dimensional model in terms of $\sigma$-dependent effective $\alpha^{*}$ and $C^{*} \equiv$ $\left(\Delta M_{s}^{2}\left|N_{y}-N_{x}\right|\right)^{*}$, compared with the simulated values. $C^{*}$ is estimated by fitting the expression for $j_{W}$ (see text) to the data in the bottom panel of Fig. 3.

\begin{tabular}{lcccccccc}
\hline \hline$\sigma\left(\mu \mathrm{m}^{-2}\right)$ & $\alpha^{*}$ & \multicolumn{1}{c}{$C^{*}\left(\mathrm{~A}^{2} / \mathrm{m}\right)$} & $j_{\text {dep }, \text { int }}^{\text {pred }}\left(\mathrm{A} / \mathrm{m}^{2}\right)$ & $j_{\text {dep }, \text { int }}^{\text {sim }}\left(\mathrm{A} / \mathrm{m}^{2}\right)$ & $H_{W}^{\text {pred }}(\mathrm{mT})$ & $H_{W}^{\text {sim }}(\mathrm{mT})$ & $v_{m}^{\text {pred }}\left(H_{W}\right)(\mathrm{m} / \mathrm{s})$ & $v_{m}^{\text {sim }}\left(H_{W}\right)(\mathrm{m} / \mathrm{s})$ \\
\hline 0 & 0.0200 & $2.92 \times 10^{-10}$ & $14 \times 10^{12}$ & $15 \times 10^{12}$ & 2.75 & 2.75 & 457 \\
1562.5 & 0.0221 & $2.52 \times 10^{-10}$ & $12.1 \times 10^{12}$ & $13 \times 10^{12}$ & 3.05 & 3.0 & 398 & 457 \\
3125 & 0.0238 & $2.45 \times 10^{-10}$ & $11.7 \times 10^{12}$ & $12 \times 10^{12}$ & 3.25 & 3.25 & 389 & 419 \\
4687.5 & 0.0258 & $2.36 \times 10^{-10}$ & $11.3 \times 10^{12}$ & $11.5 \times 10^{12}$ & 3.52 & 3.25 & 377 & 403 \\
6250 & 0.0283 & $2.28 \times 10^{-10}$ & $10.9 \times 10^{12}$ & $11 \times 10^{12}$ & 3.78 & 3.5 & 368 \\
\hline \hline
\end{tabular}

Finally we consider the role of the nonadiabatic spintransfer torque (bottom panel of Fig. 3, where the $\xi=0.04$ case is shown) on the DW dynamics. For $\xi>0$ and $\sigma=0$, there is no intrinsic pinning, and the DW propagates, preserving its internal structure with a finite velocity linearly proportional to the current density $j_{\text {ext }}$ up to a Walker breakdown current $j_{W}$. For $j_{\text {ext }}>j_{W}$, an antivortex is again nucleated and propagates across the strip width, reversing the polarity of the DW magnetization, and decreasing the average DW velocity. For larger $j_{\text {ext }}$, the velocity again increases with $j_{\text {ext }}$. Adding disorder induces a finite depinning threshold $j_{\text {dep }}(\sigma)$, and pushes the local maximum of $v_{m}$ or the Walker breakdown to higher $j_{\text {ext }}$. At the same time, $v_{m}$ at $j_{W}(\sigma)$ increases with $\sigma$. Thus, the voids are able to inhibit the antivortex entering the strip, enhancing the DW propagation and structural stability for intermediate current densities, $j_{W}(\sigma=0)<j_{\text {ext }}<j_{W}(\sigma>$ $0)$. This effect arises as the antivortex core is pushed across the strip width by the effective field terms in Eq. (1) (notice that the effect of the current is symmetric such that no antivortex displacement along the $y$ direction arises directly due to the current-see the middle panel of Fig. 2), a mechanism sensitive to the disturbances due to disorder. Again, there is no core pinning for $j_{\text {ext }}>j_{W}(\sigma)$, for the same reason as in the adiabatic $(\xi=0)$ case.

For $j_{\text {dep }}(\sigma)<j_{\text {ext }}<j_{W}(\sigma), v_{m}$ depends linearly on $j_{\text {ext }}$, and by extrapolating linear fits to the data to $j_{\text {ext }}=0$ all the lines cross at $v_{m}=0$ (not shown). Thus, we estimate effective values of the damping parameter from the slopes of these linear fits ${ }^{8}$ as within one-dimensional models ${ }^{23} v_{m} \propto(\beta / \alpha) j_{\text {ext }}$ for $j_{\text {ext }}<j_{W}$, with $\beta=\xi /\left(1+\xi^{2}\right)$. Our simulations (inset of the lower panel of Fig. 3) with different $\xi$ indicate that the data can be interpreted in terms of an effective $\alpha^{*}$ increasing with $\sigma^{8}{ }^{8}$ Also an effective $M_{s}^{*}=(1-\sigma L w / N) M_{s}$ emerges naturally. Thus we can explain our results with the one-dimensional model in terms of $\sigma$-dependent effective parameters: For instance, $j_{W}(\sigma)=4 \pi \gamma\left(M_{s}^{2} \Delta\left|N_{y}-N_{x}\right|\right)^{*} \alpha^{*} /\left(g \mu_{B} P\left|\beta-\alpha^{*}\right|\right)$, with $\Delta$ the DW width and $N_{x}$ and $N_{y}$ the demagnetizing factors, and $j_{\text {dep,int }}(\sigma) \equiv j_{W}(\sigma, \xi=0)$. ${ }^{23}$ Using the expression for $j_{W}$ and the values of $\alpha^{*}$ to estimate $C^{*} \equiv\left(\Delta M_{s}^{2} \mid N_{y}-\right.$ $\left.N_{x} \mid\right)^{*}$, the scaling of $j_{\text {dep,int }}$ with $\sigma$ can be reproduced remarkably well-see Table I. A similar analysis in the field-driven case, with $H_{W}=2 \pi \alpha^{*}\left(M_{s}\left|N_{y}-N_{x}\right|\right)^{*}$ and $v_{m}\left(H_{W}\right)=\left(\gamma \Delta^{*} / \alpha^{*}\right) H_{W},{ }^{23}$ reproduces the observed scaling of both $H_{W}$ and $v_{m}\left(H_{W}\right)$ with $\sigma$ (Table I). Notice that in our case $v_{m}\left(H_{W}\right)$ depends on $\sigma$ through the $\sigma$-dependent effective parameters, while for systems with only edge roughness $v_{m}\left(H_{W}\right)$ is independent of the amount of edge roughness. ${ }^{6}$

To summarize, we have presented a detailed analysis of the effect of disorder on the field- and current-driven transverse DW dynamics in a narrow and thin permalloy nanostrip. We have identified two fundamentally different pinning mechanisms, acting in different regimes of the DW propagation. The observation that there is no core pinning in the current-driven case whereas it dominates the field-driven dynamics for large fields highlights the different nature of the field and current drive in a way that can be observed only in the presence of disorder. In general, we have seen that the pinning mechanisms operating will depend on the details of the DW structure, and thus we expect that the core pinning mechanism is absent for systems with high perpendicular magnetocrystalline anisotropy as there is no (anti)vortex core that could get pinned, but it could play a role in the dynamics of vortex walls occurring in wider soft strips, ${ }^{8}$ possibly also for small applied fields. If only edge roughness is present, no core pinning should occur. Experiments should be performed to systematically study the scale dependence of $P_{\text {pin }}$ and $v_{\text {exp }}$. Finally, we point out that the observation that disorder tends to stabilize the DW internal structure and increase the maximum DW velocity by suppressing the Walker breakdown in the current-driven case suggests that it could be desirable to deliberately engineer disorder in the system, for instance, to replace notches to pin the DW in various technological applications. ${ }^{24}$

Stefano Zapperi is thanked for numerous interesting discussions on DW dynamics and disorder, and Mikko J. Alava for useful comments on the manuscript. We thank Luc Dupré and Daniël De Zutter for supporting this research. L.L. has been supported by the Academy of Finland through a Postdoctoral Researcher's Project (Project No. 139132) and through the Centres of Excellence Program (Project No. 251748). B.V.d.W. has been supported by the Flanders Research Foundation FWO.
${ }^{1}$ S. S. P. Parkin, M. Hayashi, and L. Thomas, Science 320, 190 (2008).

${ }^{2}$ M. Hayashi, L. Thomas, R. Moriya, C. Rettner, and S. S. P. Parkin, Science 320, 290 (2008).
${ }^{3}$ D. A. Allwood, G. Xiong, C. C. Faulkner, D. Atkinson, D. Petit, and R. P. Cowburn, Science 309, 1688 (2005).

${ }^{4}$ V. Lecomte, S. E. Barnes, J.-P. Eckmann, and T. Giamarchi, Phys. Rev. B 80, 054413 (2009). 
${ }^{5}$ G. Durin and S. Zapperi, in The Science of Hysteresis, edited by G. Bertotti and I. Mayergoyz, Vol. II (Academic, Amsterdam, 2006), pp. 181-267.

${ }^{6}$ Y. Nakatani, A. Thiaville, and J. Miltat, Nat. Mater. 2, 521 (2003).

${ }^{7}$ E. Martinez, J. Phys.: Condens. Matter 24, 024206 (2012).

${ }^{8}$ H. Min, R. D. McMichael, M. J. Donahue, J. Miltat, and M. D. Stiles, Phys. Rev. Lett. 104, 217201 (2010).

${ }^{9}$ L. Laurson, A. Mughal, G. Durin, and S. Zapperi, IEEE Trans. Magn. 46, 262 (2010).

${ }^{10}$ L. Laurson, C. Serpico, G. Durin, and S. Zapperi, J. Appl. Phys. 109, 07D345 (2011).

${ }^{11}$ G. Meier, M. Bolte, R. Eiselt, B. Krüger, D.-H. Kim, and P. Fischer, Phys. Rev. Lett. 98, 187202 (2007).

${ }^{12}$ M. Kläui, P.-O. Jubert, R. Allenspach, A. Bischof, J. A. C. Bland, G. Faini, U. Rüdiger, C. A. F. Vaz, L. Vila, and C. Vouille, Phys. Rev. Lett. 95, 026601 (2005).

${ }^{13}$ H. Tanigawa, T. Koyama, M. Bartkowiak, S. Kasai, K. Kobayashi, T. Ono, and Y. Nakatani, Phys. Rev. Lett. 101, 207203 (2008).

${ }^{14}$ X. Jiang, L. Thomas, R. Moriya, M. Hayashi, B. Bergman, C. Rettner, and S. S. P. Parkin, Nat. Commun. 1, 25 (2010).

${ }^{15}$ A. Vansteenkiste and B. Van de Wiele, J. Magn. Magn. Mater. 323, 2585 (2011).

${ }^{16}$ S. Zhang and Z. Li, Phys. Rev. Lett. 93, 127204 (2004).
${ }^{17}$ Y. Nakatani, A. Thiaville, and J. Miltat, J. Magn. Magn. Mater. 290-291, 750 (2005).

${ }^{18}$ A. Thiaville and Y. Nakatani, in Spin Dynamics in Confined Magnetic Structures III, edited by B. Hillebrands and A. Thiaville, Topics in Applied Physics, Vol. 101 (Springer, Berlin, 2006), p. 161.

${ }^{19} \mathrm{We}$ have checked that our main conclusions remain the same if instead of voids one considers small areas with half the saturation magnetization $M_{s}$, suggesting that our results are not limited to the specific kind of disorder we study here.

${ }^{20}$ To avoid any effect related to the initial acceleration and of the demagnetizing fields at the end of the wire, we actually calculated the average speed between a point at $0.5 \mu \mathrm{m}$ after the initial position and at $0.5 \mu \mathrm{m}$ before the end of the wire.

${ }^{21}$ Z. Li and S. Zhang, Phys. Rev. B 70, 024417 (2004).

${ }^{22}$ In fact, studying the true thermally activated creep motion by micromagnetic simulations is very challenging, due to the long time scales needed to observe several repeated pinning-depinning events, a requirement for reliable estimation of the DW velocities in the creep regime.

${ }^{23}$ A. Mougin, M. Cormier, J. P. Adam, P. J. Metaxas, and J. Ferre, Europhys. Lett. 78, 57007 (2007).

${ }^{24}$ M. A. Basith, S. McVitie, D. McGrouther, and J. N. Chapman, Appl. Phys. Lett. 100, 232402 (2012). 